\title{
LE JIHADISME DES FEMMES. POURQUOI ONT-ELLES CHOISI DAECH?
}

Autor@s: Fethi Benslama y Farhad Khosrokhavar.

París: Seuil, 2017.

\section{Eguzki Urteaga}

eguzki.urteaga@ehu.eus

Universidad del País Vasco - España

Recibido: 26-10-2017

Aceptado: 06-06-2018

Fethi Benslama y Farhad Khosrokhavar acaban de publicar su libro titulado Le jihadisme des femmes. Pourquoi ont-elles choisi Daech? (Le yihadismo de las mujeres. ¿Por qué han elegido Daech?) en la editorial Seuil. Conviene recordar que Benslama es psicoanalista y catedrático de psicopatología y decano de la facultad de Estudios psicoanalíticos en la Universidad París-Diderot. Entre sus obras más recientes se pueden mencionar La guerre des subjectivités en Islam (2014), L'idéal et la cruauté (2015) y Un furieux désir de sacrifice. Le surmusulman (2016). Khosrokhavar, por su parte, es sociólogo y director de estudios en la Escuela de Altos Estudios en Ciencias Sociales (EHESS) de París y director del Observatorio de la radicalización en la Fundación de la Casa de las Ciencias del Hombre (MSH). Entre sus últimas publicaciones figuran La radicalisation (2014), Prisons de France (2017) y Les juifs, les musulmans et la République (2017), escrito junto con Michel Wieviorka.

En la introducción de la presente obra, los autores recuerdan que el Estado Islámico (EI) o Daech tiene una actitud muy dura con las mujeres. "Trato desigual, encerramiento en viviendas [...] donde deben esperar a sus futuros esposos, prohibición de salir solas a la calle, imposición del velo integral, desigualdad [notable] de los derechos entre hombres y mujeres” (pg. 7). En ese contexto, ¿Cómo explicar la seducción que ejerce el Estado Islámico sobre numerosas mujeres jóvenes? ¿Cómo dar cuenta del hecho de que varios centenares de mujeres se hayan unido a Daech en Siria e Irak? Para contestar a estas preguntas, Benslama y Khosrokhavar se han unido en una colaboración entre un psicoanalista y un sociólogo. Conjuntamente desean comprender de qué manera y por qué numerosas adolescentes y jóvenes mujeres europeas se han involucrado en el yihadismo, "que se hayan unido o hayan intentado unirse a Daech, o que hayan participado u organizado acciones [violentas] en Europa o en otros lugares del mundo" (Ibídem).

“El psicoanálisis parte, a menudo, de la situación clínica de las personas o analiza los testimonios y las informaciones recogidas [en su tratamiento] de casos próximos o parecidos” (pg. 8). En general, 
la psicología de las jóvenes que se han desplazado a Oriente Medio o han intentado hacerlo se caracteriza por experimentar accidentes vitales, traumas, sufrimientos y angustias de los que han intentado escaparse alejándose de sus familias y de sus países, para, en fin de cuentas, "exiliarse de su historia [personal y] refugiarse en otro sí mismo" (pg. 9). La sociología, por su parte, intenta poner de manifiesto las condiciones sociales y culturales en las cuales se inscriben las personas. Trata de dar cuenta del modo de acción de un actor social partiendo de la sociedad en la que vive. Se esfuerza en "analizar los determinantes socioculturales, [...] económicos [y] políticos de sus acciones y de sus motivaciones" (Ibídem).

Asociando las aportaciones de ambas perspectivas, este trabajo ha consistido en formular un cierto número de articulaciones entre lo individual y lo social, a partir del análisis de "la trayectoria de jóvenes chicas o de mujeres que se han [involucrado] en [el] yihadismo" (pg. 11). Esta visión evita caer en los determinismos sociológicos y psicológicos. De hecho, "la sumisión de una joven mujer a una norma erigida en un sistema totalitario, que exige el sacrificio, solo puede comprenderse si se pone en relación con elementos psíquicos y sociales" (Ibídem). En efecto, "la radicalización se enraíza en la vida psíquica de ciertos individuos, pero también en una sociedad en el seno de la cual el cemento social parece carecer, cada vez más, de consistencia. Una sociedad sin utopía, donde puede ejercerse la seducción de 'distopías', una sociedad que [solo asigna un lugar a sus jóvenes] en términos de una adolescencia sin fin [...]. Una sociedad [...] donde ser chica o chico, mujer u hombre, se ha convertido en problemático para numerosos individuos, lo que puede convertir en atractivas [ciertas] utopías represivas y regresivas" (pg. 12).

A ese respecto, es preciso subrayar que, a diferencia de las organizaciones islamistas radicales anteriores, como Al Qaeda, "Daech ha ofrecido una concepción inédita del yihadismo de las mujeres y ha [realizado] una manipulación de los resortes propiamente femeninos de su compromiso" (pg. 10). La existencia de un territorio erigido en símbolo y encarnación de un Islam ideal contribuye al carácter atractivo de esa oferta. De hecho, 500 mujeres provenientes del Viejo Continente están hoy en día en Siria e Irak. Para comprender esa realidad, el presente estudio se basa en la producción científica de estos últimos años, así como en el estudio minucioso de unos sesenta casos que provienen, bien de la clínica psicoanalítica, bien de la reflexión desarrollada por las ciencias sociales, bien de las investigaciones empíricas llevadas a cabo por la sociología y la antropología. Los autores estiman que "las informaciones recogidas son, hoy en día, suficientemente numerosas y fundamentadas como para [poder ofrecer una visión] cualitativa general del yihadismo de las mujeres" (pg. 11).

En el primer capítulo, que desea poner de manifiesto algunas características generales de estas mujeres, los autores recuerdan que, "en 2015, según un informe confidencial de los servicios de inteligencia [galos], el número de mujeres francesas [que se ha desplazado] a Siria e Irak estaba en clara progresión con respecto al año anterior. En 2014, 150 francesas ya se encontraban en Siria al lado de los diferentes grupos yihadistas" (pg. 13). En diciembre de 2015, "sobre los 600 ciudadanos franceses presentes en las filas de la organización Estado Islámico, se hallaban 220 mujeres, frente a 
164 en septiembre del mismo año" (Ibídem). No en vano, en 2016, como consecuencia de los retrocesos territoriales del EI, tanto en Siria como en Irak, frente a las tropas de la coalición y los grupos insurgentes, y las dificultades crecientes para entrar en estos países, el flujo de las llegadas se ha ralentizado, hasta interrumpirse prácticamente en 2017.

Ante semejante panorama, "los propagandistas del EI han privilegiado las acciones yihadistas en los países de origen de las [mujeres] que querían [involucrarse] en la 'guerra santa' contra Occidente. Se han multiplicado en Europa los intentos de atentados [dirigidos a objetivos particulares] o [realizados] a ciegas" (pg. 14). Hoy en día, el principal temor de las autoridades galas concierne el posible retorno de los yihadistas y la posibilidad de que cometan atentados en Europa. Actualmente, en Francia, alrededor de 200 yihadistas vueltos de Siria han sido identificados por la policía y los servicios de inteligencia. A nivel europeo, un reciente informe de Europol (2016) indica que "el número de mujeres detenidas por actividades vinculadas al terrorismo yihadista se ha incrementado de manera exponencial desde 2013”, pasando de 6 a 128 entre 2013 y 2015 (pg. 14). Globalmente, 5.000 mujeres europeas se han desplazado a territorios ocupados por Daech.

En Francia, estas mujeres provienen mayoritariamente de las pequeñas clases medias y de las clases medias, sobre todo en el caso de las conversas que representan un tercio de estas mujeres, ya que provienen de familias cristianas, judías, agnósticas o ateas. "En su gran mayoría, no residen en [los suburbios desfavorecidos], a diferencia de la gran mayoría de los jóvenes hombres [involucrados en] Daech" (pg. 15). Según Benslama y Khosrokhavar, la aspiración al [desplazamiento a] Siria [de estas mujeres] encuentra su resorte en una visión romántica del amor, en un deseo de exotismo y de cambio de escenario, o en la aspiración de convertirse en una [verdadera] mujer" (pg. 15). Esa voluntad se expresa por el anhelo de casarse y de tener hijos precozmente (Ibídem). Asimismo, encuentra su origen "en un deseo de compromiso humanitario frente a las [atrocidades] perpetradas por [Bachar Al] Assad contra su [propio] pueblo" (Ibídem). A su vez, "la imagen del hombre ideologizado y virilizado está en el punto de mira de esta juventud femenina: es el que se expone a la muerte y se muestra viril, serio y sincero" (Ibídem). Buscan "ese marido ideal, capaz de restaurar la imagen de la masculinidad" (pg. 16).

La figura del marido ideal les permite escaparse del malestar generado por la inestabilidad y la fragilidad que caracterizan las parejas modernas. Estas mujeres están a la búsqueda de "una forma de utopía antropología donde el vínculo de confianza y la sinceridad auténtica se compaginan con una desigualdad [aceptable e incluso deseable], con una complementariedad en el seno de la pareja" (pg. 16). Las páginas Internet yihadistas del Estado Islámico exaltan "la nobleza de la mujer-madre asociada a una confianza absoluta en un hombre concebido como un héroe [que proporciona] un apoyo indefectible" (Ibídem). Reivindican un retorno al rol tradicional de la mujer en oposición a la igualdad de género promovida por las sociedades contemporáneas (Ibídem). Esta fascinación por el modelo familiar tradicional mitificado resulta de la evolución de la familia moderna, cada vez más precaria y recompuesta. 
Frente a una adolescencia y una juventud que se prolongan, en las cuales predominan la dependencia afectiva y financiera hacia los padres, numerosas jóvenes se involucran en un mundo virtual, a través de Internet. "El yihadismo que encuentran en él puede dar a las jóvenes [inmersas] en [una] crisis identitaria, la sensación de convertirse en adultas [adhiriéndose] a su causa" (pp.1718). Además, "la inestabilidad del matrimonio moderno en las familias de las cuales provienen a menudo estas chicas jóvenes, o al que se sienten expuestas en su vida futura, las conduce a considerar que la unión eterna con un combatiente de la fe llenará su aspiración a un vínculo románticamente indestructible" (pg. 18).

Más allá, lo que atrae irresistiblemente estas mujeres en el joven yihadista exaltado por la propaganda es el hecho de que afronte la muerte. "Ven en él un hombre nuevo, un super-hombre cuya violencia lo sitúa por encima [de los demás]" (Ibídem). Para estas mujeres, la visión romántica del amor se conjuga con cierta atracción por la guerra y la violencia. "Una parte de estas jóvenes chicas está, en efecto, fascinada por la violencia guerrera. Si el marido asume ese rol violento, suscriben a él y lo apoyan completamente. Algunas [practican] indirectamente la violencia” (pg. 20), mientras que otras participan directamente en la represión y encuentran en el poder represivo ejercido sobre las nomusulmanas la sensación de haberse convertido en adultas al tratarse de un rito de paso. A su vez, "la búsqueda de la autoridad a toda costa conduce estas jóvenes a buscar ardientemente el poder bajo su forma más represiva" (pg. 18). De la misma forma, "el yihadismo es elegido en razón del vacío ideológico [reinante] en el mercado del radicalismo" (pg. 19).

A la hora de desplazarse a Oriente Medio en los territorios bajo dominio del EI, "el hecho de que una chica joven parta por Siria puede invitar sus amigas a [seguir su ejemplo], dado que la competencia y la imitación juegan un rol importante en las dinámicas de grupo" (pg. 22). En el caso de las conversas, a menudo, el entorno ignora las nuevas motivaciones y creencias de la joven, lo que revela el abismo que separa los mundos juveniles y adultos. Así, "se crea un distanciamiento que puede conducir a la ruptura con la familia, en nombre de un ideal que la joven encuentra y adopta en las redes sociales, en las relaciones de complicidad con las amigas de su edad" (pp.22-23). De esa forma, algunas de estas jóvenes tienen la sensación de existir, de ser personas por sí mismas o a través de sus maridos. Lo importante, para ellas, es su voluntad de participar en un movimiento que las supera y de recuperar una identidad islámica perdida.

En el segundo capítulo, dedicado al matrimonio al estilo Daech, los autores subrayan que "el Estado Islámico instaura una relación al género que está en conformidad con la sharia. Una interpretación rígida de las leyes islámicas prevalece en principio” (pg. 25). El problema es que estas leyes mantienen una relación compleja con la estructura comunitaria, como lo demuestra el matrimonio. De hecho, el matrimonio islámico rompe con la preeminencia de la familia en las sociedades musulmanas tradicionales (Ibídem). "Las jóvenes mujeres están, lo más a menudo, reunidas en [...] unos edificios públicos situados bajo la dirección de una mujer que hace reinar en su seno, de manera a menudo rígida, unas normas vividas como agresiones [...] por [las residentes]" (Ibídem). Como lo indican Benslama y Khosrokhavar, "los jóvenes combatientes les hacen una visita 
y [las eligen] al término de una [breve conversación]. Por lo tanto, se trata de dos individuos que [contraen matrimonio] sin prácticamente conocerse” (pg. 26).

Además, la comunidad, encarnada por los padres, está completamente ausente y el matrimonio no depende de su opinión al respecto. En ese sentido, es cuestión de "una forma de compromiso entre el individualismo moderno y el Islam tradicional, que ignora la estrategia matrimonial de las familias concernidas" (pg. 26), de modo que el EI se sustituya a los padres. En otras palabras, el matrimonio amparado por Daech se caracteriza por imponer el matrimonio antes de las relaciones sexuales y por ubicarlo bajo el auspicio del Estado (pp.26-27). Una vez casadas, las relaciones de las jóvenes mujeres con sus maridos son de diferente índole. Para las que buscan el romanticismo, se hallan ante una situación paradójica. Algunas de ellas deciden emigrar para encontrarse con un hombre que han conocido a través de Internet. Pero, lo más a menudo, el amor romántico nace después del matrimonio, a pesar de que las relaciones sentimentales no tengan tiempo de profundizarse, ya que el marido es enviado al frente. Estas mujeres manifiestan, frecuentemente, un afecto idealizado por su marido muerto en mártir. Pero, la muerte no es la única razón de la separación, ya que puede resultar de una incompatibilidad (Ibídem).

En el tercer capítulo, que analiza la relación de las mujeres yihadistas con la muerte, los autores recuerdan que, "de 1981 a 2011, sobre cerca de 2.300 atentados-suicidas cometidos en el mundo, 125 habían sido [perpetrados] por mujeres, lo que representa menos del 5\% del conjunto" (pg. 31). El primer atentado-suicida cometido por una mujer en nombre del yihad islámico ha tenido lugar el 4 de octubre de 2003 en un restaurante de Haifa. Para las organizaciones que las reclutan, "representan un interés estratégico, en la medida en que están menos sospechadas de ser kamikazes y están menos vigiladas" (pg. 32). La radicalización de estas mujeres obedece, en parte, "a la misma lógica que la de los hombres: sensación de humillación, profundo resentimiento, capacidad de actuar acrecentada por una organización [...] y, por último, voluntad de humillar al humillador” (pg. 33). Además, en el caso del yihadismo, se trata de acceder a un estatus valorado y recompensado en el más allá por un lugar privilegiado en el paraíso.

En los campos de batalla, tanto en Oriente Medio como en Asia, "el compromiso de las mujeres [con los grupos] yihadistas se explica, a menudo, por la voluntad de vengar la muerte del marido, del hermano, del padre o de un miembro muy próximo a la familia" (pg. 33). Padecer los efectos de la guerra, las consecuencias del encarcelamiento de sus maridos y la presencia de las tropas de ocupación aumenta su sensación de humillación. "La muerte de las mujeres en mártires puede igualmente revestir una dimensión anti-patriarcal e incluso feminista. Se encuentra, a veces, la voluntad de alzarse al rango de los hombres mostrándose capaces de morir por una causa santa" (Ibídem). De hecho, las mujeres componen aproximadamente el $10 \%$ de la población yihadista que se ha desplazado a Siria e Irak.

Más precisamente, los autores establecen una tipología de las adolescentes y jóvenes mujeres yihadistas en su relación con la muerte. Para un primer grupo, ampliamente mayoritario, "la cuestión de la muerte no es la más inmediata ni la más importante” (pg. 35). Los autores denominan ese grupo 
"el de la muerte sálvica por procuración", ya que la muerte del marido en un acto sacrificial las eleva a un estatus de "mater dolorosa" (pg. 36). Un segundo grupo minoritario de mujeres desea combatir con las armas en la mano, pero no están autorizadas a hacerlo por el Estado Islámico. Se habla, a su propósito, de "sobre-musulmanas" o de "heroínas negativas", que no se confunden entre sí (pg. 36). Un tercer grupo de jóvenes mujeres puede ser descrito como totalmente desconectado de la realidad mientras se encuentran en Europa. Desean vivir un romanticismo revolucionario (Ibídem). Las califican de "románticas [alejadas] de la realidad". "Buscan sobre todo la ruptura con [una vida diaria taciturna] privada de un horizonte de esperanza" (pg. 37). El cuarto grupo es el de las jóvenes mujeres traumatizadas que buscan huir de su trauma yendo muy lejos: "dan una dimensión peregrinante a su voluntad de olvidar su trauma causado por una violencia real o imaginaria en el seno de la familia" (Ibídem). Se trata de "fugitivas del trauma" (Ibídem).

Estas mujeres son muy conscientes, en general, "de la naturaleza de su compromiso, de la eventualidad de la muerte [de su] marido y de la necesidad en la que se encontrarían de vivir su viudedad solas o, después de algunos meses, en compañía de un nuevo marido" (Ibídem). La muerte de sus maridos es vivida, frecuentemente, por estas mujeres como "un reparto de tareas. Es también una manera de reconocerse y de distinguirse como mujer con respecto al hombre" (pg. 38). Su afirmación como mujeres pasa por la maternidad, ya que desean ser madres y criar a sus hijos. A su vez, privilegian una sociabilidad femenina y consideran el hogar como su ámbito de predilección. Por lo cual, rechazan cualquier participación de los hombres en las tareas domésticas que viven como una “intrusión” (Ibídem). La vida que les propone Daech realiza esa aspiración. Durante la ausencia del marido, pueden gozar de la sociabilidad femenina, disfrutar de cierta autonomía, educar a sus hijos, vivir a su antojo y cultivar su jardín secreto. En ese sentido, manifiestan cierta nostalgia hacia la familia patriarcal (pg. 39).

Para estas mujeres, el hombre es el padre de familia que combate en el exterior, que protege a la mujer y que puede morir repentinamente. Esto no impide la descendencia sino todo lo contrario, puesto que es preciso "asegurar la perpetuación del linaje al servicio del [islamismo] yihadista" (pg. 40). En esta perspectiva, la concepción y la continuidad de la familia en el tiempo parecen estar desconectadas. Los autores distinguen tres momentos en la actitud de las mujeres, como madres, ante la muerte (pg. 41):

1) antes del desplazamiento a Siria, están impregnadas por un imaginario que está alejado de la realidad del Medio Oriente;

2) después de su instalación en Siria, su matrimonio y su maternidad, se enfrentan a la muerte de su marido en el campo de batalla;

3) con el transcurso del tiempo, se produce una desilusión, e incluso un rechazo del sistema represivo, aunque pueda producirse igualmente una radicalización aún superior y una identificación total al Califato. 
- En el primer momento, el imaginario de la maternidad, que se conjuga al futuro, da un sentido a la vida presente y la posible muerte del marido aparece como algo abstracto. Prevalece el ideal de una vida marcada por el romanticismo, el exotismo y la excitación que genera la novedad.

- Una vez instaladas, si algunas pierden sus ilusiones, muchas se casan, tienen hijos y pierden sus maridos, bien en el frente, bien como consecuencia de bombardeos. A veces, expresan el deseo de seguir los pasos de sus maridos muertos como mártires.

- Tras varios meses de duelo, algunas mujeres aceptan casarse de nuevo. "Ese segundo matrimonio [tiene] un sentido más o menos idéntico al primero. [...] La mujer debe contemplar la posibilidad de la muerte de su segundo marido", lo que resulta complejo y doloroso (pg. 43). Pero, si ciertas mujeres se adaptan a la vida en Siria bajo el EI, otras pierden sus esperanzas y desean volver a Europa, sin renunciar por ello a sus creencias religiosas.

"La muerte, para la mayoría de las jóvenes mujeres que adhieren a Daech, es vivida, en el mejor de los casos, por procuración" (pg. 44). No en vano, algunas mujeres, a menudo jóvenes, afirman su voluntad de "luchar contra las fuerzas del mal" (Ibídem). En Siria, "aprenden el manejo de las armas y asumen responsabilidades en la represión de las mujeres y el sometimiento de la sociedad civil a las normas islámicas represivas. Aprenden también a fabricar explosivos" (Ibídem). En general, no temen herir o matar al enemigo designado. A ese respecto, los autores distinguen la "sobre-musulmana" y la "heroína negativa":

- La primera adopta la ideología de un islamismo identitario muy fundamentalista que la dota de un habitus coherente y de una concepción de sí misma que se afirma en una forma de existencia distinta e incluso en tensión con la sociedad a fin de encontrar un nuevo sentido.

- La segunda va más allá de la simple afirmación de sí misma y desea someter a los demás a través del uso de la fuerza, apropiándose los rasgos esenciales de los yihadistas masculinos.

En Europa, una parte de las chicas y jóvenes mujeres que no han podido desplazarse a Oriente Medio han optado por la lógica de la violencia y han intentado cometer atentados. Si entre las "sobremusulmanas" la relación al odio es distante, ya que lo que prevalece es la relación con el marido combatiente que hace la guerra contra los infieles y encarna la seriedad y la confianza, las "heroínas negativas" recurren a la violencia porque rechazan la sociedad que es identificada al mal (pg. 46).

Los autores observan, además, que algunas adolescentes y jóvenes mujeres, en una actitud infantil, "yihadizan" su trauma vinculándolo con las violencias padecidas en el seno de la familia o con la sensación de haber sido violentadas (pg. 47). El viaje o el sueño del viaje a Siria reflejan la aspiración a olvidar ese trauma. El matrimonio aparece entonces como una huida hacia adelante. No buscan afrontar el problema sino rodearlo ocultándolo a través del exilio. De hecho, "en numerosas familias [pertenecientes a] las clases populares [de los suburbios desfavorecidos], pero también de las clases medias urbanas, de las que provienen muchos jóvenes hombres yihadistas así como algunas 
mujeres, la violencia es algo cotidiano e inexorable" (Ibídem). Los casos estudiados por los autores muestran cómo "la violencia y la ausencia de autoridad [paterna], así como la delincuencia de los demás miembros de la familia, tienen efectos traumáticos sobre las [menores]” (pg. 51). De hecho, algunas chicas dicen haber padecido violencias físicas e incluso violaciones.

Para terminar ese apartado, Benslama y Khosrokhavar constatan que el universo psíquico de las adolescentes difiere del de las adultas. En el caso de estas últimas, la relación con la ideología está mucho más articulada. "La justificación de la acción violenta se inscribe en una visión del mundo en la cual el Islam está perseguido por los cruzados [...]. La liberación pasa, [por lo tanto], por el ejercicio de la violencia contra los que deniegan a los musulmanes la [dotación] de un sistema político y cultural en el cual la religión de Alá jugaría un rol clave” (pg. 52). Las adolescentes y posadolescentes, en cambio, pueden ejercer la violencia sin la menor inhibición. Los factores efectivos adquieren una importancia superior. Mientras que unas han padecido violencias durante su infancia, otras mantienen una actitud transgresora hacia la sociedad y desean afirmarse de manera frontal manteniendo una actitud provocadora.

En el cuarto capítulo, que estudia la relación de las mujeres yihadistas con lo religioso, los autores observan que "las religiones revisten un significado diferente según se trata de mujeres adultas o de adolescentes y posadolescentes" (pg. 55). No en vano, en ambos casos, "lo religioso se reduce a lo sagrado" (Ibídem). Es un mundo imaginario que da un sentido al Islam, que se halla desprovisto de cualquier anclaje histórico y social. Para estas jóvenes mujeres, lo religioso se declina en varios registros. Uno de los más importantes es el del neo-salafismo. En ese caso, desean ser mujeres integrales, mujeres totales, mujeres ante todo. Ser sobre todo mujeres "encuentra su sentido, según esta visión regresiva de la feminidad, en un Islam puro y duro, que define la identidad femenina como distinta de la del hombre y promueve la segregación como una medida de higiene social” (pg. 56). La separación de los universos masculinos y femeninos conforta estas mujeres neo-salafistas en su propia identidad. Por ejemplo, el velo integral sustrae la mujer de la mirada de los hombres y, más allá, del espacio público y del mundo común.

Otro sentido de lo religioso para ciertas mujeres yihadistas es "la reacción ante una laicidad convertida en intolerante que postula que cualquier mujer velada es una fundamentalista” (pg. 57). La voluntad de transgresión ante una laicidad represiva, que pretende prohibir el velo en la Universidad e incluso en la calle, toma una forma radical en algunas jóvenes mujeres. Así, el fundamentalismo musulmán se nutre de la intolerancia laica. Otras mujeres se adhieren al islamismo radical para crear un sagrado que se opondría a una modernidad securalizada. "Persiguen la búsqueda de una transcendencia represiva" (pg. 58). En ese sentido, "las mujeres yihadistas interiorizan la utopía regresiva de la mujer pura" (pg. 59), reaccionando contra un individualismo reducido al consumismo.

El quinto capítulo, consagrado a la moral sexual de las mujeres yihadistas, los autores observan que, lo que llama la atención en las mujeres que se han radicalizado es la conciencia moral severa que las caracteriza "que precede la elección de la religión bajo sus formas salafistas o rigoristas. A menudo, [la adolescencia] está marcada [...] por unos tormentos vinculados con [las] vivencias de una 
sexualidad condenable [desde el punto de vista de la moral religiosa]" (pg. 61). En ese sentido, el giro rigorista permite "atenuar el sufrimiento moral y la culpabilidad muy viva que sienten, conformándose a lo que es interpretado como el deseo de Dios" (Ibídem). Esta actitud es aún más evidente cuando se trata de jóvenes mujeres que han padecido violencias sexuales por sus padres o sus allegados, y parecen ser numerosas entre las radicalizadas. "El caos provocado por los traumas las ha [...] conducido a encontrar en el control religioso una puesta en orden saludable" (Ibídem).

Numerosas jóvenes mujeres de confesión musulmana están a la búsqueda de un matrimonio con un hombre "muy musulmán" que les permita frenar sus pulsiones y les imponga unos límites draconianos. "El marido yihadista garantizaría que permanezcan en el [marco] virtuoso de la ley [...]. Es el goce amenazante que [confiere] su fuerza al rigor moral” (pg. 62). Es la razón por la cual estas mujeres consienten vivir bajo el régimen de la coacción y consideran esta última como una libertad, ya que "están liberadas del tormento de un goce transgresor y peligroso" (Ibídem). No solamente esta servidumbre voluntaria les procura la tranquilidad del cuerpo y del alma, sino que, además, experimentan el "placer de la servidumbre" (pg. 63). La búsqueda de un marido según el ideal islámico puede conducir estas mujeres a sacrificar sus vínculos con sus familias de origen, a convertirse en implacables con las demás mujeres y a usar formas de seducción contrarias a la moral de la que se reclaman para llegar a sus fines. Se produce, igualmente, una escalada represiva en ciertas mujeres, puesto que, cuando más se someten al rigorismo, más severas son con las demás mujeres, sobre todo cuando su entorno se opone a su elección religiosa.

El tránsito al universo moral rigorista tiene un nombre y un estatus en el islamismo yihadista: el arrepentimiento, "sobre-interpretado en el sentido de la intolerancia e incluso de la violencia" (pg. 65). Este consiste en una experimentación de remordimientos, vergüenzas y expiaciones, en una atmosfera mórbida cargada de culpa y de auto-castigo (pg. 65). En esta óptica, dichas mujeres manifiestan, a menudo, un temor de la transparencia. Para ellas, el velo toma el significado de "una apropiación de su propio cuerpo, percibido como preocupante a causa de los signos enigmáticos de goce de la que es [objeto], pero, igualmente, como extraño, a través de las miradas masculinas que lo cosifican" (pg. 67). "Se sienten físicamente cernidas por las miradas que las convierten en totalmente visibles, captivas de una exterioridad expoliadora" (Ibídem). A su entender, el velo tendría una función liberadora al proceder a un encerramiento que les devuelve su intimidad y dignidad.

En otros casos, el detonante del arrepentimiento es una ruptura o una decepción sentimental que conduce la persona a una transformación radical de su estilo de vida, "pasando de una vida [considerada] disoluta al rigorismo más [absoluto] y a la búsqueda apremiante de un matrimonio con un salafista o, más radicalmente, un yihadista que conforte la mujer y la proteja de sus propios temores transgresivos" (pg. 68). El cambio puede ser repentino o progresivo, en un itinerario tumultuoso hecho de alternancias entre periodos de silencio total y de castidad integral. En muchos casos, la aparición de una conciencia moral severa, que se traduce por la adhesión al islamismo radical, se produce tras un rechazo de la persona por el mundo exterior que provoca una fuerte angustia, "vinculada a la percepción de un rechazo interior de un disfrute reprobado" (pg. 69). 
A veces, "la adopción de un [discurso] salafista por mujeres que han conocido la prostitución, la toxicomanía [o] la delincuencia, les permite purificarse [y] 'virginizarse' [moralmente]” (Ibídem). De ese modo, la culpabilidad y el auto-castigo aparecen frecuentemente en las mujeres que se han comprometido en el yihadismo. En otros casos, la conversión es una forma de salvarse y de alejarse del suicidio, "transformándolo en un deseo de martirio" (pg. 71). Esto demuestra que "el compromiso religioso de muchas mujeres que se radicalizan tiene como terreno de combate su [propio] cuerpo y como enemigo su deseo percibido como culpable. Encuentran en la ideología salafista un envoltorio inmunizante y un arma para luchar contra sus propios demonios" (pg. 72).

En el sexto capítulo, que se interesa por la relación de estas mujeres con la religión y lo sagrado, Benslama y Khosrokhavar constatan que el yihadismo de las mujeres permite acceder a la esencia de la ideología yihadista, "que no deriva simplemente del Islam como religión, [sino también] de un llamamiento a un sagrado que excede la religión como institución” (pg. 75). De hecho, el ámbito de lo sagrado en el Islam tiende a estar vinculado con la prohibición. Lo sagrado, asociado al cuerpo, a la sexualidad y al espacio propio, supone una puesta a distancia y una imposibilidad de tocar (pg. 78). La intocabilidad tiene un triple sentido:

1) la prohibición de entrar en contacto físicamente con el cuerpo;

2) la imposibilidad asociada a la inmunidad, al respeto, a la salud y a la salvación;

3) la intocabilidad entendida como la puesta a cubierto.

En cuanto a la santidad, pertenece al registro de la pureza absoluta y de la veneración reservada a Dios y a su palabra.

En general, las conversas radicalizadas desconocen las categorías y los matices de la teología musulmana, "lo que ha permitido al fundamentalismo difundir una religiosidad de la santa ignorancia" (pg. 80). El islamismo salafista "está saturado de sacralidades arcaicas. Es [muy] identitario [y] es profuso en marcajes corporales y en exteriorizaciones de signos religiosos" (pp.80-81). Para las conversas y las reconvertidas, descendientes de inmigrantes provenientes de países de confesión musulmana, "esta sobre-sacralización aporta una solución rápida a numerosos casos de impases en la transmisión, de trastornos en la desafiliación, de herencias [marcadas] por el rechazo de su exilio por ciertos padres, que viven la inmigración como un [pecado], y, en ciertos casos, como una damnación" (pg. 81). Algunas chicas y jóvenes mujeres se sienten obligadas de reparar ese pecado original a través de su exilio a territorios controlados por el Estado Islámico. "La reparación se [acompaña] de vergüenza cuando se le añaden las heridas de la relegación urbana y de la segregación [social]” (Ibídem).

En el séptimo capítulo, centrado en la sacralización de la mujer, los autores indican que "es [el objetivo] principal del salafismo y de su utilización guerrera por el yihadismo. Consiste en su naturalización integral, [asimilando] el sexo al género, operación inversa [a su] diferenciación moderna y a su proyecto de emancipación. La naturalización [se realiza] a través de la precipitación 
en la maternidad" (pg. 85). La mujer es consagrada como madre y es separada del espacio público. Es reseñable, al respecto, que, sobre las sesenta mujeres estudiadas, más del tercio ha padecido sufrimientos vinculados con sus madres al tratarse de madres que las han abandonado o maltratado, de madres odiosas de la feminidad o de madres "fusionales" (Ibídem). Los trastornos psíquicos causados a estas mujeres por sus madres son especialmente devastadores. Para ellas, "la maternidad les parece una escapatoria saludable, que restaura su [autoestima] y les permite desprenderse del [narcisismo] de su madre, de su influencia o de su [ausencia]" (pg. 86).

La sacralización de las mujeres, que pasa por el hecho de convertir el cuerpo en tabú, afecta especialmente a las adolescentes. Implica la prohibición de tocar y de ser tocada, de ver y de ser vista. "La obsesión de la pureza que la [acompaña] pretende circunscribir un cuerpo que se ha convertido en molesto, a causa de las transformaciones juveniles que [experimenta], las cuales son fuente de tumulto pulsional, de desasosiego y de culpabilidad" (pg. 89). La sacralización permite, asimismo, a la adolescente "deshacerse de la imbricación familiar que le inspira [...] [cierta] aversión e incluso horror" (pg. 90).

En el octavo y último capítulo, centrado en la figura paterna entre las mujeres yihadistas, Benslama y Khosrokhavar subrayan que la ausencia del padre en las familias de las candidatas al yihad explica parcialmente el debilitamiento de la autoridad y la búsqueda de figuras tutelares y represivas a través de la adhesión al yihadismo. A menudo, más que un padre ausente, prevalece un padre negligente o malvado, lo que afecta a la atención y al cuidado simbólico que debe garantizar un progenitor a su hija, inscribiéndola "en una cadena de generaciones y en un deseo de que no sea anónima” (pg. 93). Si se computa el número de padres que han infringido la ley y que han huido de sus obligaciones familiares, se constata que constituyen la mayoría de las mujeres comprometidas en el yihadismo. En la mayoría de los desplazamientos a Siria e Irak, las jóvenes mujeres no se dirigen a sus padres sino a sus madres con las cuales mantienen contactos. "La piedad de la madre [y] la promesa de salvarla en el más allá, contrastan con el silencio sobre el padre” (pg. 94).

Al término de la lectura del libro Le jihadisme des femmes. Pourquoi ont-elles choisi Daech?, es obvio reconocer la originalidad del objeto de estudio, ya que muy pocas investigaciones han sido llevadas a cabo y escasas reflexiones han sido desarrolladas sobre el perfil y las motivaciones de las mujeres yihadistas. Estas son analizadas gracias a una perspectiva híbrida que integra las aportaciones de la sociología y del psicoanálisis, lo que permite tener, a la vez, una visión macro, meso y micro de ese colectivo. A su vez, la presente obra asocia un perfecto conocimiento de la literatura científica existente a nivel mundial sobre ese tema y un estudio minucioso de 60 casos de mujeres yihadistas, lo que constituye una muestra a tener en cuenta dado la dificultad de obtener información fiable y contrastada sobre ese fenómeno en alza estos últimos años. Los autores consiguen, asimismo, compaginar una sistematización teórica, un rigor analítico y una fundamentación empírica. Por último, combinan una extrema densidad con un estilo fluido, lo que propicia la lectura de la obra. La única pena es que el libro no sea más extenso, lo que hubiese permitido abordar aspectos adicionales como el retorno de estas mujeres a Europa y su proceso de reinserción social. 
Desde una perspectiva de género, además de interesarse por las mujeres yihaditas, al considerarlas como un objeto de estudio pertinente científicamente para mejorar nuestra comprensión del yihadismo isalamista, los autores consideran a estas mujeres como sujetos activos, dotados de reflexividad y de motivaciones ideológicas, capaces de realizar elecciones estratégicas y acciones individuales (incluso violentas), y no como meras acompañantes de sus conyugues o meras víctimas pasivas del contexto internacional o de la dominación masculina (Bourdieu, 2000). A su vez, ponen de manifiesto la desorientación identitaria de ciertas jóvenes mujeres ante la inestabilidad y la fragilidad de las familias modernas, por una parte, y, el debilitamiento y/o la menor identificación de los roles tradicionales, así como la recomposición incierta e individualizada de los mismos, por otra parte. Esta situación las conduce a buscar un modelo donde los roles de los hombres y de las mujeres estén claramente diferenciados, aunque sean contrarios a su emancipación. Asimismo, en su intento por conciliar las perspectivas sociológica y psicoanalítica, y aunque aludan a las motivaciones feministas de ciertas combatientes yihadistas, Benslama y Khosrokhavar descuidan las aportaciones realizadas por los gender studies a la comprensión de la representación de la mujer inmigrante en la cultura patriarcal (Suárez Villegas, 2013).

En cualquier caso, la lectura de esta obra es altamente recomendable al tratarse de una investigación pionera llevada a cabo por dos especialistas internacionalmente reconocidos de ese tema.

\section{Bibliografía}

Benslama, Fethi (2014): La guerre des subjectivités en Islam. París: Lignes. : (2015) (dir.): L'idéal et la cruauté. París: Lignes. . (2016): Un furieux désir de sacrifice. Le surmusulman. París: Seuil.

Benslama, Fethi y Khosrokhavar, Farhad (2007): Le jihadisme des femmes. Pourquoi ont-elles choisi Daech? París: Seuil.

Bourdieu, Pierre (2000): La dominación masculina. Barcelona: Anagrama.

EUROPOL (2016): European Union Terrorism Situation and Trend Report, julio de 2016.

Khosrokhavar, Farhad (2014): La radicalisation. París: Editions de la MSH. : (2017): Prisons de France. París: Robert Laffont.

Khosrokhavar, Farhad y Wieviorka, Michel (2017): Les juifs, les musulmans et la République. París: Robert Laffont.

Suárez Villegas, Juan Carlos (2013): "La mujer inmigrante en la cultura patriarcal y su reflejo en los medios de comunicación". En: Sphera publica: revista de ciencias sociales y de la comunicación, vol.1, nº.13, pp.77-94. 